\title{
Can Indocyanine Green Angiography be Used for Measuring One Arm-Brain Circulation Time?
}

\author{
Sujoy Banik Lakshmi Venkatraghavan ${ }^{1}$ \\ ${ }^{1}$ Department of Anesthesia, Toronto Western Hospital, University \\ Health Network, University of Toronto, Toronto, Ontario, Canada
}

J Neuroanaesthesiol Crit Care 2018;5:37-38.

The measurement of one arm-brain circulation time is usually considered as the time taken for unconsciousness, after injection of bolus dose of intravenous anesthetic agents. However, arm-brain circulation time varies with many factors such as lipid solubility, pharmacokinetic profile, circulatory volume and cardiac output. Some patients, especially the elderly and those with low cardiac output states, may have a longer arm-brain circulation time and hence during induction, additional boluses of anesthetic agents need to be delayed to avoid adverse hemodynamic consequences. Digital subtraction angiography (DSA) is the gold standard for the measurement of cerebral circulation time; ${ }^{1,2}$ however, this may not be logistically feasible, and the method of injection of dye differs from that of anesthetic induction.

Indocyanine green (ICG) videoangiography is increasingly used during cerebrovascular surgery to visualise the cerebral vasculature to confirm optimal placement of aneurysmal clips and to assess the patency of intracranial bypasses, potentially obviating the need for intraoperative DSA. ${ }^{3}$ ICG is inert, relatively safe as it avoids contrast exposure, quick, reliable and provides real time information. ICG provides an immediate visual cue to the presence of the dye in the cerebral vasculature, and therefore, the arm-brain circulation time may be estimated as the time taken from injection of the drug till the first appearance of visualisation of contrast in the microscope screen.

We analyzed data from a previously collected database of another IRB approved study where 36 consecutive adult patients, 18 to 50-year-old, the American Society of Anaesthesiologists Grade I-III, undergoing intracranial surgery required ICG videoangiography intraoperatively. Patients were excluded if they refused consent, were pregnant, allergic to ICG or had significant liver or kidney dysfunction. Anesthesia management was standardised according to institutional protocol. In all patients, $7.5 \mathrm{mg}$ of ICG dye diluted to a total volume of $10 \mathrm{ml}$ was administered through the right forearm intravenous line (18-gauge) over $10 \mathrm{~s}$ followed by $10 \mathrm{~mL}$ of $0.9 \%$ normal saline flush while the operating

\begin{abstract}
Address for correspondence Lakshmi Venkatraghavan, MBBS, MD, DNB, FRCA, FRCPC, Department of Anesthesia, Toronto Western Hospital, University Health Network, University of Toronto, McL 2-405, Toronto, Ontario, Canada (e-mail: lakshmi.venkatraghavan@uhn.ca).
\end{abstract}

microscope primed to fluorescence mode. The median time taken to first appearance of contrast visualisation was $21 \mathrm{~s}$ (range: 17-26 s). Among them, 13 patients undergoing extracranial-intracranial bypass surgery for Moyamoya disease had longer median time (26 s, range 23-31 s), than subset of patients undergoing arteriovenous malformation excision ( $n=11$, median $17 \mathrm{~s}$, range: $14-19 \mathrm{~s}$ ) and those with intracranial aneurysm clipping $(n=12$, median time $22 \mathrm{~s}$, range $18-25 \mathrm{~s}$ ). No perioperative adverse events were noted with dye injection.

Our pilot data confirms that arm-brain circulation time ranges from 17 to $26 \mathrm{~s}$ as previously reported. ${ }^{4}$ Our study finding may have an important implication during induction of anesthesia, which needs confirmation by a larger trial; the non-exclusion of low cardiac output states may also be a limitation of this study. Patients with arteriovenous malformations have the fastest anesthetic induction due to shortest arm-brain circulation time. In contrast, patients with intracranial steno-occlusive diseases have long arm-brain circulation time and hence, the anesthesiologist need to wait for at least $30 \mathrm{~s}$ after the initial bolus before administering further doses of anesthetic agents so that inadvertent hypotension can be avoided.

\section{Funding \\ None.}

\section{Conflict of interest}

None.

\section{References}

1. Kim MN, Durduran T, Frangos S, Edlow BL, Buckley EM, Moss HE, et al. Noninvasive measurement of cerebral blood flow and blood oxygenation using near-infrared and diffuse correlation spectroscopies in critically brain-injured adults. Neurocrit Care 2010;12:173-180.

2. Liu X, Czosnyka M, Donnelly J, Budohoski KP, Varsos GV, Nasr $\mathrm{N}$, et al. Comparison of frequency and time domain methods of assessment of cerebral autoregulation in traumatic brain injury. J Cereb Blood Flow Metab 2015;35:248-256.
DOI https://doi.org/ 10.1055/s-0037-1618322 ISSN 2348-0548.
Copyright $\odot 2018$ Indian Society of Neuroanaesthesiology and Critical Care
License terms

()ㅇㅇ $\Theta \circledast$ 
3. Doss VT, Goyal N, Humphries W, Hoit D, Arthur A, Elijovich L, et al. Comparison of intraoperative indocyanine green angiography and digital subtraction angiography for clipping of intracranial aneurysms. Interv Neurol 2015;3:129-134.
4. Stoelting RK, Miller RD. Intravenous anesthetics. In: Miller RD, Stoelting RK, eds. Basics of Anesthesia. 4th ed. New York: Churchill-Livingstone; 2000:58-69. 